\title{
O uso de substâncias psicoativas e o estado mental: avaliando a função cognitiva através do Mini Exame do Estado Mental
}

The use of psychoactive substances and the mental status: assessing the cognitive function through the Mini Mental State Examination

El uso de sustancias psicoactivas y el estado mental: evaluación de la función cognitiva a través del Mini Examen del Estado Mental

João Pedro Satuf Silva de Carvalho ${ }^{1 *}$, Carina Gabriela Andrade Oliveira ${ }^{1}$, Carlos Alberto Pereira Pinto .

\section{RESUMO}

Objetivo: Avaliar os possíveis efeitos na esfera cognitiva de pacientes usuários de entorpecentes em tratamento no Centro de Atenção Psicossocial - Álcool e Drogas (CAPS AD) de Betim tendo como parâmetro a aplicação do teste do Mini Exame do Estado Mental (MEEM). Métodos: Trata-se de uma pesquisa quantitativa, exploratória, descritiva, baseada em questionário semiestruturado. Foram entrevistados, no período de março a novembro de 2019, 68 indivíduos; sendo 62 homens e 6 mulheres, com idades entre 22 e 69 anos, que utilizaram os serviços proporcionados pelo CAPS AD durante a coleta dos dados. Resultados: Os resultados obtidos após aplicação e interpretação dos questionários demonstram suspeita de deterioração cognitiva nos usuários de drogas, sendo que apenas 13,24\% dos pacientes apresentaram resultados adequados no MEEM. Dentre as funções cognitivas avaliadas durante a aplicação do teste, a memória, foi a que apresentou pior resultado. Conclusão: O uso excessivo de substâncias entorpecentes pode ocasionar danos neurológicos severos. As prováveis deteriorações cognitivas apresentadas remontam necessidades de mais estratégias de prevenção (rodas de conversa, dinâmicas, oficinas de memória) e de existências de centros de reabilitação, tal como o CAPS AD de Betim.

Palavras-chave: Cognição, Alcoolismo, Transtornos relacionados a substâncias.

\begin{abstract}
Objective: To evaluate the possible effects on the cognitive sphere of patients using narcotics undergoing treatment at Centro de Atenção Psicossocial - Álcool e Drogas (CAPS AD) Betim having as a parameter the application of the Mini Mental State Examination (MMSE) test. Methods: This is a quantitative, exploratory, descriptive research based on a semi-structured questionnaire. From March to November 2019, 68 individuals were interviewed; 62 men and 06 women, aged 22 to 69 years, who used the services provided by CAPS AD during data collection. Results: The results obtained after application and interpretation of the questionnaires show suspicion of cognitive deterioration in drug users, and only $13.24 \%$ of patients had adequate MMSE results. Among the cognitive functions evaluated during the test application, memory presented the worst result. Conclusion: Excessive use of narcotic substances can cause severe neurological damage. The probable cognitive impairments presented date back to the need for more prevention strategies (conversation circles, dynamics, memory workshops) and the existence of rehabilitation centers, such as CAPS AD de Betim.
\end{abstract}

Keywords: Cognition, Alcoholism, Substance-related disorders.

\section{RESUMEN}

Objetivo: Evaluar los posibles efectos en la esfera cognitiva de los pacientes que usan narcóticos sometidos a tratamiento en Centro de Atenção Psicossocial - Álcool e Drogas (CAPS AD) Betim teniendo como parámetro la aplicación de la prueba Mini Mental State Examination (MMSE). Métodos: Esta es una investigación cuantitativa, exploratoria, descriptiva basada en un cuestionario semiestructurado. De marzo a noviembre de 2019, se entrevistó a 68 personas; 62 hombres y 06 mujeres, de 22 a 69 años, que utilizaron los servicios proporcionados por CAPS AD durante la recopilación de datos. Resultados: Los resultados obtenidos después de la aplicación e interpretación de los cuestionarios muestran sospecha de deterioro

1Pontifícia Universidade Católica de Minas Gerais, Betim - MG. *E-mail: joaopedrosatuf@gmail.com

PUBLICADO EM: 2/2021 
cognitivo en los narcóticos dependientes, y solo el $13.24 \%$ de los pacientes tuvieron resultados MMSE adecuados. Entre las funciones cognitivas evaluadas durante la aplicación de la prueba, la memoria presentó el peor resultado. Conclusión: El uso excesivo de sustancias narcóticas puede causar daño neurológico severo. Las probables deficiencias cognitivas presentadas se remontan a la necesidad de más estrategias de prevención y la existencia de centros de rehabilitación.

Palabras clave: Cognición, Alcoholismo, Trastornos relacionados con sustancias.

\section{INTRODUÇÃO}

O consumo de drogas está crescendo gradativamente, e isso pode ser explicado pelas diversas mudanças em valores e costumes pós-modernos. De acordo com a Organização Mundial da Saúde - OMS- cerca de $6 \%$ da população mundial possui algum tipo de transtorno psiquiátrico grave devido ao uso de substancias alcoólicas e outras drogas. Essa porcentagem possui grande significância, podendo implicar em prejuízos no âmbito social, judiciário, psicológico e econômico, constituindo-se num grave problema de saúde pública (LARENGIS CP E MAGGI A, 2012).

O município de Betim, localizado na região metropolitana de Belo Horizonte, Minas Gerais, apresenta progressão no tratamento da dependência química nos moldes preconizados pelas diretrizes da Reforma Psiquiátrica através do Centro de Atenção Psicossocial - Álcool e Drogas (CAPS AD) (BETIM, 2017). O Centro de Atenção Psicossocial Álcool e Outras Drogas (CAPS-AD) desempenham um importante papel de ordenar a rede de atendimento aos usuários de álcool e outras drogas em seu território de atuação estimulando a articulação entre os dispositivos comunitários sociais e de saúde para permitir a atenção integral e inclusão social dos usuários e seus acompanhantes (MOURA FG e SANTOS JE, 2011).

Ademais, o CAPS ou Núcleo de Atenção Psicossocial se caracteriza como um serviço de saúde aberto e comunitário do Sistema Único de Saúde (SUS). Ele é um lugar de referência e tratamento para pacientes psiquiátricos acometidos por transtornos mentais, psicoses, neuroses graves e demais quadros, cuja intensidade e/ou persistência justifiquem sua permanência em um local de cuidado intensivo, comunitário, inserido na comunidade, promotor de saúde e qualidade de vida. O objetivo principal dos CAPS é fornecer atendimento para toda a população que ele abrange, por meio do acompanhamento longitudinal e da recapacitação social de seus usuários, através do incentivo ao trabalho e lazer, ao fortalecimento dos laços familiares e comunitários e o exercício dos direitos civis (LARENTIS CP e MAGGI A, 2012).

No que diz respeito às drogas, estas podem ser definidas como substâncias não produzidas pelo organismo que têm a propriedade de agir no cérebro modificando funções mentais, como o julgamento, 0 humor, a percepção e o comportamento de maneira geral. Suas funções são múltiplas e podem estar ligadas ao desejo do homem de buscar formas de alterar seu estado de consciência, explorar suas emoções, melhorar seu estado de espírito, intensificar a sensação dos sentidos. (MELO JRF e MACIEL SC, 2016).

Entretanto, a prevalência de déficits na saúde mental, juntamente com uso de drogas, é uma relação problemática; pois, o uso de substâncias é capaz de promover no corpo do indivíduo uma série de efeitos negativos, devido ao uso crônico, e a dependência química (SMITH LL, et al., 2017).

Um dos aspectos que deve ser destacado é que o uso contínuo de qualquer substância psicoativa produz uma doença cerebral em decorrência de seu uso inicialmente voluntário. A consequência é que, a partir do momento que a pessoa desenvolve uma dependência, o uso passa a ser compulsivo destruindo muitas das melhores qualidades do indivíduo o que pode contribuir para desestabilização dele com a sua família e com a sociedade (DUALIBI S, et al., 2011).

Em comparação com a população geral, dependentes químicos possuem maior dificuldade em realizar tarefas que utilizam funções executivas e planejamento, assim como atividades que necessitem de controle de impulso ou formação de conceitos. Isso pode ser explicado pelos vários prejuízos neuropsicológicos que o uso agudo ou crônico de substâncias psicotrópicas causam, como por exemplo, o bradipsiquismo; a dificuldade em processar informações; os prejuízos na praxia construtiva e na percepção visioespacial; a diminuição da capacidade de planejar e organizar tarefas, o detrimento da abstração; o retardo na 
aprendizagem e realização de funções executivas; a perda de memória operacional e episódica e por fim a lentificação do tempo de reação. Pacientes que já apresentaram algum desses prejuízos causados por drogas ilícitas relataram, em pesquisa, que sentem falta de motivação para mudança de seus hábitos de vida. Tal fato pode ser corroborado pelas alterações nas capacidades de planejar e organizar tarefas, acarretando na dificuldade de tomar decisões assertivas sobre a manutenção de seu tratamento, afetando a sua aderência e eficácia. (FERREIRA VRT e COLOGNESE BT, 2014).

Baseado nesse contexto, pesquisar sobre a cognição humana, torna-se fundamental para a compreensão dos efeitos cognitivos e emocionais das substâncias psicoativas. O uso crônico de álcool é frequentemente associado à prejuízos nas áreas motora e cognitiva, assim como, têm-se demonstrado que este uso provoca alterações no desempenho de determinadas funções cerebrais. A principal área de cognição que necessita de uma avaliação mais padronizada e abrangente nesses pacientes etilistas de longa data é a memória, sendo que esta consiste no significado que cada organismo registra frente a exposição à eventos ou experiências (RIGONI MS, et al., 2013).

Assim, mais da metade dos indivíduos que se apresentam para o tratamento de alcoolismo demonstram de leve a severo déficit no raciocínio abstrato, funções executivas, habilidades viso espaciais, novos aprendizados e memória (OLIVEIRA MS, et al., 2015). Estudos mostram que o uso crônico do álcool pode provocar uma série de prejuízos nas funções neurocognitivas, podendo ser correlacionadas com problemas de aprendizagem, abstração, resolução de problemas, análise e síntese visuoespacial, velocidade psicomotora, velocidade do processamento de informações e eficiência cognitiva (RIGONI MS, et al., 2013). Em virtude disso, é necessário realizar um rastreamento cognitivo.

Atualmente, o mini exame do estado mental (MEEM) consiste em um questionário para pessoas adultas e idosos com o intuito de rastrear problemas na função cognitiva. O teste já foi traduzido para mais de 35 idiomas e, possivelmente, é o instrumento de avaliação mais citado na história da saúde mental (MELO DM e GONÇALVES BAJ, 2015).

A análise desse trabalho consiste em avaliar os possíveis efeitos na esfera cognitiva de pacientes usuários de entorpecentes em tratamento no CAPS AD da cidade de Betim, no período compreendido entre de 12 de março de 2019 a 30 de novembro de 2019 , tendo como parâmetro/ instrumento a aplicação do teste do MEEM.

\section{MÉTODOS}

Esta pesquisa caracteriza-se como um modelo de estudo exploratório, quantitativo e descritivo. Esse tipo de estudo possui como objetivo descrever as características de determinada população ou fenômeno e, ainda, o estabelecimento de relações entre as variáveis (MINAYO MCS, et al., 2010).

Foi utilizado um questionário semiestruturado, aplicado face a face aos entrevistados, com duração média de cerca de oito minutos. Como formas de registro foram utilizadas cópias impressas do teste preconizado (ORTEGA FJR, 2014). Os dados da pesquisa foram individualmente coletados na área externa da própria instituição (CAPS AD) de modo a seguirmos um roteiro de perguntas previamente estabelecidas.

Atrelado a isso foi apresentado um termo de consentimento livre e esclarecido para os entrevistados, que foram contatados, de forma voluntária, através de uma parceria com o CAPS AD que nos proporcionou 0 contato com os usuários. A execução da pesquisa ocorreu no período de março a novembro de 2019. O grupo pesquisado foi composto por pacientes cuja faixa etária era maior de 18 anos de idade com capacidade de comunicação e de entendimento verbal que usufruíam, no período da coleta dos dados, as atividades médicas e terapêuticas proporcionadas pelo CAPS AD de Betim.

Além disso foram excluídos da amostra pacientes com alguma psicopatologia prévia que poderia interferir nos resultados obtidos do MEEM devido algum déficit cognitivo apresentado, como por exemplo, a esquizofrenia. Outros critérios utilizados de exclusão foram: pacientes com quadros de demências, deficientes auditivos e / ou verbais e / ou visuais. A coleta de dados foi realizada por intermédio da aplicação do MEEM o qual é constituído de duas partes. A primeira abrange orientação temporal espacial, registros, atenção e cálculo, com pontuação máxima de 21 pontos. Já a segunda aborda habilidades específicas, tais como 
capacidade de nomeação, de obediência a um comando verbal e a um escrito de redação livre de uma sentença e de cópia de um desenho complexo com pontuação máxima de 9 pontos, totalizando um escore de 30 pontos (FERNÁNDEZ-SERRANO MJ, et al., 2011).

O MEEM equivale a um instrumento que possui o seu próprio método de análise dos resultados, sendo considerado adequado: pontuação acima de 27 pontos e inadequado: pontuação menor ou igual a 24 pontos. Em caso de menos de 4 anos de escolaridade, o ponto de corte passa para 17, ao invés de 24 (FERNÁNDEZSERRANO MJ, et al., 2011).

O grupo de pesquisadores (composto de graduandos do curso de medicina e por um orientador) antes de iniciar a execução da pesquisa, apresentou o projeto para equipe multiprofissional do CAPS AD a fim de propor melhor articulação com esses profissionais. Foi agendado uma reunião a qual teve participação da gerente do CAPS AD de Betim, psicólogos, médico, referência técnica de saúde, terapeuta ocupacional; dentre outros membros que opinaram e nos auxiliaram na melhor maneira de iniciar abordagem com os pacientes.

Paralelamente a isso, os resultados que apresentaram pontuações (conforme a escolaridade do sujeito) abaixo do esperado foram comunicados primeiramente ao serviço do CAPS AD e, juntamente com a equipe, foram planejadas estratégias que aperfeiçoaram o tratamento dos trabalhadores junto aos usuários. Como exemplos dessas atividades implementadas e, com participação satisfatória dos pacientes, podemos incluir jogos, oficinas de memória e rodas de conversa nas quais eram debatidos temáticas e questionamentos levantados pelos próprios usuários dos serviços da referida Instituição.

Importante ressaltar que a pesquisa obedeceu às normas contidas na resolução 466-12 do Conselho Nacional de Saúde que dispõe sobre pesquisas envolvendo seres humanos. Também, antes da aplicação do questionário foi entregue uma via do termo de consentimento livre e esclarecido (TCLE) o qual fornecia informações de todos os procedimentos por meio de uma linguagem objetiva e clara.

Foi solicitada a assinatura desse termo a todos os 68 participantes que aceitaram participar da entrevista de forma livre e espontânea. O projeto foi submetido ao Comitê de Ética e Pesquisa da Pontifícia Universidade de Minas Gerais Betim sob o parecer número 3.103.545 e na Secretaria Municipal de Saúde de Betim sob o parecer número 3.270.117.

\section{RESULTADOS}

A amostra oriunda dessa pesquisa foi composta por 68 participantes, sendo 62 homens e 6 mulheres, cujas faixas etárias oscilaram entre 22 e 69 anos. O nível de escolaridade predominante foi de 4 a 8 anos, com 35 pacientes, seguido de 25 pacientes com mais de 8 anos de estudo e, por fim, 8 pacientes com 0 a 3 anos de escolaridade.

Foi avaliado o desempenho das funções cognitivas que compõem o MEEM conforme a comparação do que seria esperado do entrevistado de acordo com a sua escolaridade. No campo da atenção e cálculo nenhuma escolaridade atingiu a pontuação média necessária, (+ de 8 anos: 2,44; 4 a 8 anos: 2,26; 0 a 3 anos: 1), sendo que a faixa escolar de 0 a 3 anos apresentou o pior resultado. Esta função cognitiva demonstrou maior déficit nos pacientes do que todas as outras funções (Tabela 1).

No campo da memória de evocação nenhuma escolaridade atingiu a pontuação média necessária $(0$ a 3 anos: 1,88; 4 a 8 anos: 1,60; + de 8 anos: 1,56); sendo que a faixa escolar de + de 8 anos apresentou o pior resultado. Esta função cognitiva foi a segunda função mais deficitária entre os pacientes, já que apresenta melhor resultado apenas ao se comparar com o campo da atenção e cálculo.

No campo da linguagem apenas a escolaridade de 0 a 3 anos não atingiu a pontuação média necessária, (0 a 3 anos:7,63; 4 a 8 anos: 7,94; + de 8 anos :8,4).

No campo da orientação temporal espacial nenhuma escolaridade atingiu a pontuação média necessária, ( 0 a 3 anos: 7,75; 4 a 8 anos: 7,37; + de 8 anos: 8,20), sendo a escolaridade de 4 a 8 anos com o pior resultado em comparação com o esperado. 
No campo de registros todas as escolaridades apresentaram a pontuação média necessária ( 0 a 3 anos: 3; 4 a 8 anos: 2,94; + de 8 anos: 3), sendo a escolaridade de 4 a 8 anos com o pior resultado em comparação com o esperado.

Tabela 1 - Analisando o desempenho das funções cognitivas de acordo com a escolaridade do paciente e das médias obtidas.

\begin{tabular}{lccc}
\hline Função cognitiva & Escolaridade & Média obtida & Média esperada \\
\hline \multirow{2}{*}{ Atenção e cálculo } & 0 a 3 anos & 1,0 & 4,15 \\
& 4 a 8 anos & 2,26 & 4,4 \\
& + de 8 anos & 2,44 & 4,66 \\
\hline \multirow{2}{*}{ Memória de evocação } & 0 a 3 anos & 1,88 & 2,5 \\
& 4 a 8 anos & 1,6 & 2,64 \\
& + de 8 anos & 1,56 & 2,8 \\
\hline \multirow{2}{*}{ Linguagem } & 0 a 3 anos & 7,63 & 7,5 \\
& 4 a 8 anos & 7,94 & 7,94 \\
& + de 8 anos & 8,4 & 8,4 \\
\hline \multirow{2}{*}{ Orientação temporal e espacial } & 0 a 3 anos & 7,75 & 8,3 \\
& 4 a 8 anos & 7,37 & 8,8 \\
\multirow{2}{*}{ Registro } & + de 8 anos & 8,2 & 9,3 \\
& 0 a 3 anos & 3 & 2,5 \\
& 4 a 8 anos & 2,94 & 2,64 \\
\hline
\end{tabular}

Fonte: Carvalho JPSS, et al., 2020.

Apenas $13,24 \%$ dos entrevistados apresentaram resultados adequados no MEEM de acordo com sua escolaridade (Tabela 2).

Tabela 2 - Resultados obtidos após aplicação dos questionários.

\begin{tabular}{cc}
\hline Resultado Adequado & Resultado Inadequado \\
\hline $13,24 \%$ & $86,76 \%$ \\
\hline Fonte: Carvalho JPSS,
\end{tabular}

Dos pacientes entrevistados apenas 16,18\% faziam uso crônico de apenas drogas ilícitas, $70,59 \%$ faziam uso de apenas álcool e 13,24\% faziam o uso concomitante das duas substâncias. Além disso, ainda em conformidade com a tabela 03 , a média da pontuação dos pacientes que usavam apenas drogas foi de 23,45 , a daqueles que usavam álcool e drogas foi de 22,67 e a daqueles que utilizavam apenas álcool foi de 22,63 (Tabela 3).

Tabela 3 - Análise do percentual e das médias obtidas de acordo com tipo de entorpecente utilizado pelo entrevistado.

\begin{tabular}{ccc}
\hline Entorpecente & Percentual & Médias obtidas \\
\hline Apenas droga & $16,18 \%$ & 23,45 \\
Apenas álcool & $70,59 \%$ & 22,63 \\
Álcool e drogas & $13,24 \%$ & 22,67 \\
\hline
\end{tabular}

Fonte: Carvalho JPSS, et al., 2020.

\section{DISCUSSÃO}

Foi observado, a partir da interpretação dos dados obtidos, que a maioria dos usuários entrevistados do CAPS AD de Betim apresentou baixa/média escolaridade. Tal fato já é referenciado na literatura como um grave problema, decorrente, muitas vezes, do próprio uso da droga. O início do consumo na maior parte dos casos é iniciado precocemente o que favorece evasão escolar desde cedo. Jovens usuários de substâncias 
abandonam o ambiente escolar, não somente para fazer o uso da droga; mas, sobretudo motivados pelo baixo desempenho e pela dificuldade de aprendizado oriunda dos prejuízos cognitivos desencadeados pelo uso abusivo da droga (SCHEFFER M, et al., 2010).

Do total de 68 pacientes entrevistados, apenas 13,24\% apresentou resultados adequados no MEEM de acordo com sua escolaridade, o que pode demonstrar que existe uma relação do uso crônico de álcool e drogas com a demência precoce. O álcool pode provocar lesões difusas no cérebro, prejudicando, além da memória, capacidade de julgamento, de abstração e de comportamento. As características clínicas de comprometimento cerebral associado ao uso do álcool variam de perdas cognitivas leves até danos graves que produzem demência (DUALIBI S, et al., 2011).

Os resultados obtidos na pesquisa demonstraram que os usuários de álcool e de drogas participantes da pesquisa apresentaram um pior resultado nos campos da atenção/cálculo, da memória de evocação e da orientação tempo/espacial. Tal fato corrobora com a hipótese de que o uso crônico de álcool e drogas afete mais diretamente, regiões cerebrais pré-frontais e temporais e o prosencéfalo, áreas responsáveis pelos pensamentos abstratos e planejamento, associações de pensamentos e lembranças, atenção, fluência verbal, memória visual, memória verbal, capacidade de aprendizagem e funções executivas (CUNHA PJ, et al., 2010).

No campo da linguagem apenas a escolaridade de 0 a 3 anos não atingiu a pontuação média necessária. No campo de registros todas as escolaridades apresentaram a pontuação média necessária, sendo a escolaridade de 4 a 8 anos com o pior resultado em comparação com o esperado, o que pode ser devido ao seu maior número de participantes. $O$ fato de nenhuma escolaridade ter se apresentado inadequada corrobora com a hipótese de que o uso crônico de álcool e drogas não interfere de maneira significativa na capacidade do paciente em realizar a função de registro e de linguagem (SCHEFFER M, et al., 2010).

Dos pacientes entrevistados apenas 16,18\% faziam uso crônico de apenas drogas ilícitas, $70,59 \%$ faziam uso de apenas álcool e 13,24\% faziam o uso concomitante das duas substâncias. Juntamente com isso a média da pontuação dos pacientes que usavam apenas drogas foi de 23,45 , a daqueles que usavam álcool e drogas foi de 22,67 e a daqueles que utilizavam apenas álcool foi de 22,63. Ambos esses achados corroboram com o fato de que a demência gerada pelo uso crônico de álcool é muito mais severa do que a gerada pelo uso de drogas devido à presença das comorbidades Wernick e Korsakoff em sua abstinência; porém deve ser levado em consideração que a escolaridade não foi considerada nesta divisão, podendo gerar viés de pontuação nesta categoria (ISENBERG-GRZEDA E, et al., 2012).

A síndrome de Wernicke-Korsakoff é composta por um grupo de sinais e sintomas neuropsiquiátricos causados pela deficiência nutricional de tiamina (vitamina B1). O principal fator responsável pela deficiência dessa vitamina é o consumo abusivo de álcool etílico. Tal enfermidade se apresenta em duas fases de um mesmo processo patológico: inicialmente surge, mas nem sempre, a encefalopatia de Wernicke (fase aguda da síndrome) que consiste em: estado confusional agudo, oftalmoparesia, ataxia (perda da coordenação motora) e nistagmo (não tão comum) (ISENBERG-GRZEDA E, et al., 2012).

Além disso, com a cronificação do processo patológico, a encefalopatia pode progredir para um quadro permanente (síndrome de Korsakoff) caracterizado por uma amnésia anterógrada e confabulação, à medida que os sinais da primeira desaparecem. Caso a identificação e o tratamento dessa síndrome sejam tardios ou ineficazes, poderá surgir estupor, coma e, eventualmente, a morte (SILVA A e ENES A, 2013).

O consumo de droga, lícitas ou ilícitas, é um problema social que atinge o mundo todo afetando pessoas de diversas faixas etárias e proporciona consequências graves à sociedade, já que o uso contínuo destas substâncias leva a quebra de relações sociais e familiares, com prejuízos cognitivos, ocupacionais, aumento nos acidentes e na criminalidade culminando em perda de vidas humanas e de capacidade produtiva de muitas pessoas (SCHLINDWEIN-ZANINI R e SOTILI M, 2019).

A principal fortaleza do trabalho é sua contribuição para endossar estudos sobre possíveis malefícios para saúde mental decorrente do uso abusivo de entorpecentes. Também, a importância do tema abordado reside na necessidade de se discutir o real efeito que a dependência de álcool e de outras drogas acarreta o 
funcionamento cognitivo dos pacientes e, por consequência, nos demais âmbitos sociais e familiares. Isso porque o consumo de droga, lícitas ou ilícitas, é um problema social que atinge o mundo todo afetando pessoas de diversas faixas etárias e trazendo repercussões graves à sociedade (SCHLINDWEIN-ZANINI R e SOTILI M, 2019). Além disso, a detecção de alterações no cognitivo de pacientes usuários de drogas pode permitir uma reflexão sobre o tratamento oferecido a esses indivíduos. Dessa forma pode-se aprimorá-lo, somando-o com estratégias de reforço das áreas testadas pelo minimental, de forma a atrasar e/ou eliminar o déficit encontrado (SCHLINDWEIN-ZANINI R e SOTILI M, 2019).

\section{CONCLUSÃO}

Este trabalho mostrou-se um aliado em uma demanda solicitada pela sociedade de políticas públicas de enfrentamento ao uso abusivo de drogas, assim como possibilitou uma resposta da correlação do uso crônico de drogas e a deterioração da capacidade cognitiva do usuário. Com base nisso, espera-se que os resultados encontrados neste estudo venham a contribuir para ampliar os conhecimentos existentes na área, de modo a incentivar a realização de novas pesquisas. Cabe a sugestão de inserir sistematicamente atividades que visem protelar os danos causados pelo uso de substâncias na cognição dos pacientes ou até mesmo aprimorar as suas funções cognitivas nos serviços de saúde mental.

\section{REFERÊNCIAS}

1. BETIM (MG). In: Panorama demográfico, Estruturas de distribuição etária da população; Rede: atenção à saúde das pessoas com condições crônicas. Plano municipal de saúde de Betim 2018-2021. Betim: Secretaria municipal de saúde, 2017.

2. CRUZ BF, et al. Associações entre déficits cognitivos e qualidade de vida na esquizofrenia. Rev Psiq Clín. 2010; 37(5): 233-9.

3. CUNHA PJ, et al. Decision-Making Deficits Linked to Real-life Social Dysfunction in Crack Cocaine-Dependent. The American Journal on Addictions, 2010; 20: 78-86.

4. DUAILIBI S, et al Dependencia Química: prevenção, tratamento e políticas públicas. Porto Alegre: Artmed; 2011.

5. FERREIRA VRT, COLOGNESE BT. Prejuízos de funções executivas em usuários de cocaína e crack: case studies. Aval. psicol. 2014; 13 (2): 195-201.

6. FERNÁNDEZ-SERRANO MJ, et al. What are the specific vs. generalized effects of drugs of abuse on neuropsychological performance? Neuroscience \& Biobehavioral Reviews 2011, 35 (3): 377-406

7. ISENBERG-GRZEDA E, et al. Wernicke-Korsakoff-Syndrome: Under-Recognized and Under-Treated. Psychosomatics, 2012; 53(6), 507-516.

8. LARENTIS CP, MAGGI A. Centros de Atenção Psicossocial Álcool e Drogas e a Psicologia. Aletheia. 2012; (37): 121-32.

9. MELO JRF, MACIEL SC. Representação Social do Usuário de Drogas na Perspectiva de Dependentes Químicos. Psicol. cienc. Prof, 2016; 36(1): 76-87.

10. MELO DM, GONÇALVES BAJ. O uso do Mini-Exame do Estado Mental em pesquisas com idosos no Brasil: uma revisão sistemática. Ciênc. saúde coletiva, 2015; 20(12): 3865-76.

11. MINAYO MCS, et al. Pesquisa social: teoria, método, criatividade. 29 ed. Petrópolis: Vozes, 2010.

12. MOURA FG, SANTOS JE. O cuidado aos usuários de um centro de atenção psicossocial álcool e drogas: uma visão do sujeito coletivo. SMAD, Rev. Eletrônica Saúde Mental Álcool Drog. (E̋d. port.), Ribeirão Preto. $2011 ; 7$ (3): 126 32.

13. RIGONI MS, et al. Neuropsychological performance and demographic characteristics in alcoholic patients in treatment. Adicciones, 2014; 26 (3): 221-229

14. ORTEGA FJR. Manual do usuario: Mini-Examen Cognoscitivo (MEC). Edición 1. Valencia: General ASDE; 2014.

15. RIGONI MS, et al. Alcoolismo e Avaliação de Funções Executivas: Uma Revisão Sistemática Psico. 2013 ; 44 (1): 122-129.

16. SCHEFFER M, et al. Dependência de álcool, cocaína e crack e transtornos psiquiátricos. Psic.: Teor. e Pesq. 2010; 26(3): 533-41.

17. SCHLINDWEIN-ZANINI R, SOTILI M. Uso de drogas, repercussões e intervenções neuropsicológicas. Cadernos Brasileiros de Saúde Mental, 2019; 11(28): 94-116.

18. SILVA A, ENES A. Síndrome de Wernicke-Korsakoff: revisão literária da sua base neuroanatómica. Arq Med, 2013; 27(3): 121-27.

19. SMITH LL, et al. Exploring the Link Between Substance Use and Mental Health Status: What Can We Learn from the Self-medication Theory? J Health Care Poor Underserved. 2017; 28(2S): 113-31.

20. VASAN S, KUMAR A. Wernicke Encephalopathy. In: StatPearls. Treasure Island (FL): StatPearls Publishing; 2020. 